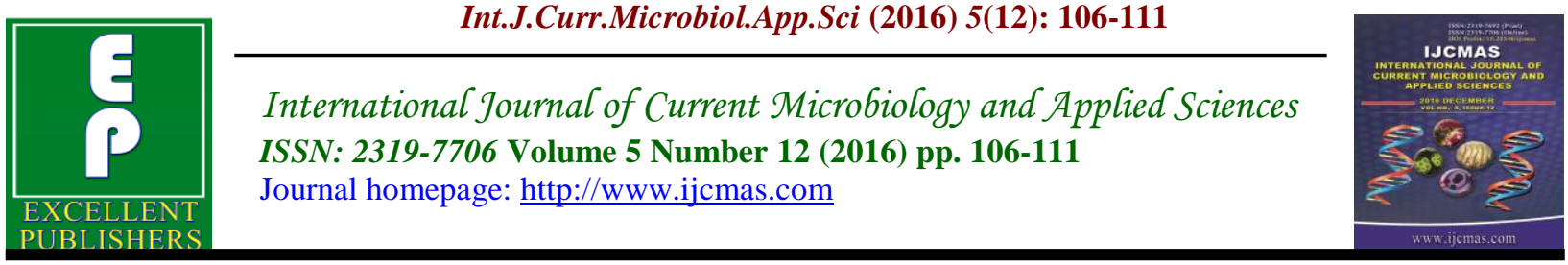

Original Research Article

http://dx.doi.org/10.20546/ijcmas.2016.512.012

\title{
Sero-Prevalence of Dengue Infection by Rapid Immunochromatographic Assay from Tertiary Care Medical College Hospital in Coimbatore, India
}

\author{
K. Gnana Prakash ${ }^{1}$, R. Someshwaran ${ }^{2 *}$ and Anbu N. Aravazhi ${ }^{3}$ \\ Department of Microbiology, Karpagam Faculty of Medical Sciences and Research, \\ Othakkalmandapam, Coimbatore-641032, India \\ *Corresponding author
}

Keywords

NS1 antigenemia, $\operatorname{IgM}$,

IgG antibody , Acute Dengue, Complications, RCT, Screening.

\section{Article Info}

Accepted:

08 November 2016

Available Online:

10 December 2016

\section{A B S T R A C T}

Dengue infection is an acute febrile endemic viral disease mostly prevalent in the tropical countries. The serological diagnosis of primary dengue infection is made by detection of NS1 antigen which is important for early (Day 1-7 of fever) and accurate diagnosis of dengue. This study is aimed to evaluate the prevalence of dengue infections by using Rapid Immuno-Chromatographic card Test detecting NS1 Antigen, Anti-IgM and Anti-IgG DENV antibodies among clinically suspected Dengue virus infections from patients attending a tertiary care hospital. This study was conducted in the form of Cross sectional study where total of 1469 serum samples received in serology section with working diagnosis of Dengue fever or other viral illnesses were subjected to Dengue virus screening for NS1, Anti-IgM and Anti-IgG Antibodies in serum was done by using Rapid Immunochromatographic Card Tests. Out of 452 clinically diagnosed dengue fever cases, 32 (7.08\%) were positive for Dengue NS1 antigen alone. And the prevalence of NS1 Antigenemia was found to be $7.08 \%$ that is 32 out of 452 clinically diagnosed Dengue fever cases in this current study. The prevalence of Acute Dengue virus infections was found to be $8.85 \%$. Rapid Immuno chromatographic card tests are simple, feasible, cost-effective, less time consuming, with less labor involved, where sensitivity and specificity are good, but this card tests are only a screening test and so it cannot be used as a stand-alone test for diagnostic confirmation. , but it could be supplemented with another diagnostic assays like ELISA for Dengue IgM and IgM, Indirect Immuno fluorescence and Polymerase Chain Reaction for appropriate diagnosis of DENV infection.

\section{Introduction}

Dengue infection is an acute febrile endemic viral disease mostly prevalent in the tropical countries (Gupta et al., 2006; Bharaj et al., 2008). The causative agent for Dengue is an arthropod-borne RNA virus (ARBO) namely Dengue virus (DENV) belonging to the Family Flaviviridae. The mechanical vector for transmitting Dengue is the mosquito mainly Aedes aegypti. The diagnosis of primary dengue infection is made by detection of NS1 antigen which is important for early (Day 1-7 of fever) and accurate diagnosis of dengue. Also the detection of IgM Anti-DENV and Anti-IgG DENV 
antibodies identifies and acute and chronic dengue infection in the individuals. This study is aimed to study the prevalence of dengue infections by using Rapid ImmunoChromatographic card Test (ICT) detecting NS1 Antigen, Anti-IgM and Anti-IgG DENV antibodies among clinically suspected Dengue virus infections from patients attending a tertiary care hospital. DENV infection is the most common febrile illness of viral etiology causing morbidity and mortality especially among children which necessitates hospitalization. Four distinct serotypes (Gubler, 1998) have been described for DENV namely Type1, Type2, Type 3 and Type4. Primary infection with any one of the DENV serotype can lead to a self-limiting illness or Dengue fever (DF). A secondary dengue infection may occur by infection with another serotype and might result in severe complications (Monath et al., 1994) namely febrile seizures, dehydration, Dengue Hemorrhagic Fever (DHF), Dengue Shock Syndrome (DSS), Hemolytic Uremic Syndrome (HUS), Acute renal failure and if left untreated Death may follow. Classical Dengue fever also known as Break bone fever has an acute onset with high grade fever with double spike (Saddle back) on and off for 4-10 days (Sathish et $a l ., 2002$ ) (Incubation period) following the bite of infected mosquito. Plethora of clinical manifestation of Dengue infection or fever are headache, muscle pain, joint pain, bleeding manifestations, maculopapular rash, loss of appetite, Hepatomegaly, Lymphadenopathy and low platelet count. Hall mark features of Dengue hemorhagic fever and Dengue Shock Syndrome other than that of Dengue fever are confluent petechial rash, Gastro-intestinal bleeding, circulatory collapse, conjunctival injection, Splenomegaly and etc. Case fatality rate among complicated Dengue could $\geq 10 \%$ but with prompt early detection and treatment the fatality rate decreases to $\leq 1 \%$ of cases.
Laboratory diagnosis of Dengue fever can be established by the following tests namely a) Viral isolation from serum or autopsy specimen; b) fourfold rise or decrease in titer in reciprocal $\operatorname{IgG}$ and $\operatorname{IgM}$ DENV antibodies from paired serum samples ie., acute and convalescent sera; c) Viral antigen demonstration by ELISA, Immunohistochemistry or by Immunofluorescence in serum, autopsied tissue or in cerebro-spinal fluid (CSF); d) Molecular detection of Dengue viral genome by PCR in serum, CSF or Autopsy samples. NS1 Antigen can be detected from approximately Day 1 to Day 7 of fever. Antibody response to infection differs from person to person according to the immune status of the host. In primary infection, IgM antibodies become detectable in about 5-6 days after onset of the disease and persist for about 2-3 months. When viral load decrease in the blood, anti-IgM level raises quickly to reach peak in about two weeks. Anti-IgG production will be at a lower level when compared to IgM. Anti$\mathrm{IgG}$ antibodies are generally detectable at low levels in about 11-12 days and increases slowly but remain detectable after several months, probably even for life. During Secondary infection IgM antibodies become detectable in about 4-5 days but the titers are lower than that of primary infection. The anti-IgG antibody level raises quickly reaching peak in about two weeks after the onset of symptoms and may persists for years. Anti-IgG DENV antibody production and a weak Anti-IgM response are characteristics of Secondary DENV infection. The sensitivity, specificity, Positive predictive value (PPV) and Negative predictive value (NPV) of Immuno-chromatographic card test are $81.8 \%, \quad 87.5 \%, \quad 75.0 \%$ and $66.6 \%$ respectively. Dengue virus mainly affects children and can result in significant morbidity requiring hospitalization. The complications of Dengue are not so 
uncommon viz., Dengue Hemorrhagic Fever and Dengue Shock Syndrome. As Dengue epidemics are common in South India especially in Tamil Nadu, Kerala and Andhra Pradesh etc. Early detection and therapy is mandatory for to manage dengue infections and its complications for which many serological test, culture isolation and molecular methods are available for prompt diagnosis. Study reports have highlighted improved performance of Immuno Chromatographic card tests (ICT) between acute and convalescent sera and necessity of paired serum samples is warranted for accurate diagnosis of Dengue infections. In this study, we had evaluated the utility of ICT to diagnose dengue among suspected patients to detect the presence of NS1 antigen, $\operatorname{IgM}$ and $\operatorname{IgG}$ DENV antibodies to diagnose Dengue fever among clinically suspected Dengue patients.

To detect the prevalence of Dengue infection or fever by using Rapid Immunochromatographic Card test among clinically suspected Dengue cases.

\section{Materials and Methods}

\section{Study Design}

This study was conducted in the form of Cross sectional study after obtaining IHEC clearance of the institution following informed written consent from patients. The study locale is Diagnostic Microbiology Section of Central Service Laboratory (CSL), Karpagam Medical College Hospital, Affiliated to Karpagam Faculty of Medical Sciences \& Research, Othakalmandapam, Coimbatore. Study population comprises Inpatients and Out- patients clinically suspected to have viral fever and or dengue fever attending Medicine, Surgery, Orthopedics, Osbtetrics \& Gynecology Department OPD. Patient details like clinical history, diagnosis and treatment details was also taken into account and analyzed. A total of 1469 serum samples received in serology section with working diagnosis of Dengue fever or other viral illnesses were subjected to Dengue virus screening for NS1, Anti-IgM and Anti-IgG Antibodies in serum was done by using Rapid Immuno-chromatographic Card Tests. The serum samples of patients were obtained from ICU, IMCU, Wards and Out Patient Clinics of various departments during the study period. The data from Patient case sheet and Serology Lab register (Diagnostic Microbiology) was compared and were analyzed for interpretation of study results. Dengue virus screening for NS1, Anti-IgM and Anti-IgG Antibodies in serum was done by using Rapid Immunochromatographic Card Test results (Dengue Day 1 test, J. Mitra \& Co., Pvt. Ltd., New Delhi) and results noted. According to this commercial kit, the sensitivity and specificity for NS1 antigen (Guzman et al., 1996; Young et al., 2000) are 100\% and $99.94 \%$, whereas the sensitivity and specificity for $\operatorname{IgM}$ and $\operatorname{IgG}$ Anti-DENV antibodies with this kit are $100 \%$ and $99.88 \%$. Serum or plasma samples were used for this test. If not tested immediately, serum was refrigerated at $2-8{ }^{\circ} \mathrm{C}$. Dengue NS1 Antigen tests were performed using $100 \mu \mathrm{L}$ serum (3 drops) and $10 \mu \mathrm{L}$ serum were used for Dengue IgM / IgG antibody detection based on kit protocol and results interpreted as 'Reactive' or 'Non-reactive' only after 20 minutes.

\section{Inclusion criteria}

Age group: 18 years to 80 years.

\section{Exclusion criteria}

Clinically suspected or confirmed bacterial, parasitic and fungal causes of fever were excluded 
Lipemic, hemolysed and bacterially contaminated serum samples.

\section{Results and Discussion}

A total of 1469 patients were included in the study were tested for the presence of DENV SpecificNS1, IgG and IgM antibodies by using RCT from August $1^{\text {st }} 2012$ to July $31^{\text {st }}$ 2015. Among 1469, $452(30.79 \%)$ cases were positive for Dengue or other forms of viral fever. And out of 452 positive cases, 32 (7.08\%) were positive for Dengue NS1 antigen alone. In 452 cases, some cases were positive for both NS1 antigen and IgM in $0.88 \%(4 / 452)$ in the Figure 1. Only 4 out of 452 cases $(0.88 \%)$ cases were positive for
Dengue IgM antibody, 44 out of 452 $(9.73 \%)$ cases were positive for $\mathrm{IgG}$ alone. In this study, shows, 450 are classical dengue fever cases and 2 cases are dengue hemorrhagic fever. The prevalence of Acute Dengue virus infections was found to be $8.85 \%$ (40 out of 452) cases were positive for Acute Dengue infection, indicates with NS1 alone, IgM alone and NS1 with IgM positivity. IgG alone in this study were 44 cases out of $452(9.73 \%)$ which indicates chronic or past Dengue infection. No Cases were found to be both IgM \& IgG Positive among the study population. And the prevalence of NS1 Antigenemia was found to be $7.08 \%$ that is 32 out of 452 clinically diagnosed Dengue fever cases in this study.

Fig.1

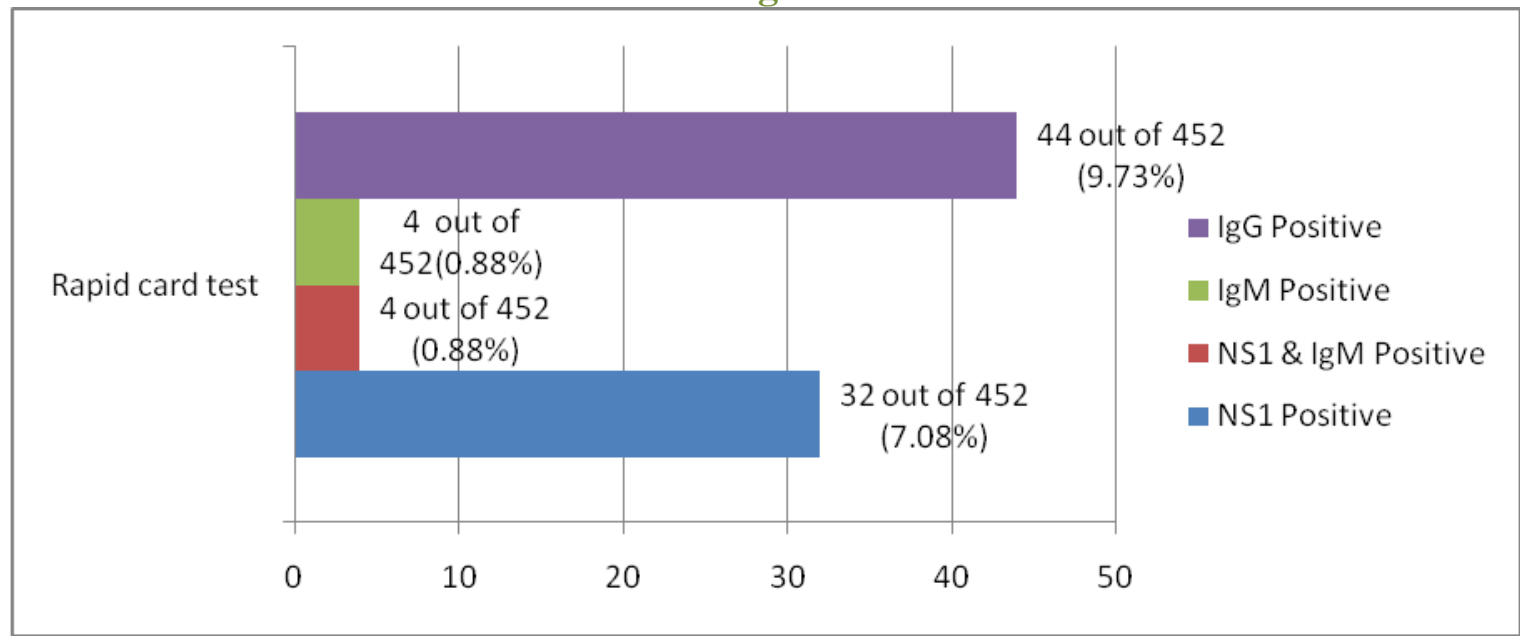

Dengue viruses have been isolated in India for first time in the year 1960s (Vijayakumar et al., 2005; Singh et al., 2005). Dengue has become an emerging disease in India particular concerned to Tamil Nadu and also other parts of the country where outbreaks of DF or DHF have occurred frequently (Cherian et al., 1994; Blacksell et al., 2006). In this study, by using Rapid card tests Acute and Chronic Dengue infections with or without recurrence were detected by Comparison of Dengue IgM, IgG antibodies and NS1 Ag. Acute Dengue Virus Infection can be detected by NS1 Ag positivity, NS1 plus Dengue IgM positivity. Similarly Chronic Dengue Infection or Past infection with DENV could also be detected by using IgG Positivity alone. This study was embarked upon to determine the utility of a rapid device in the detection of DENV antibodies in a Emergency at Tertiary care Hospitals. Many studies have been performed to evaluate rapid ICT for the detection of $\mathrm{IgM}$ and $\mathrm{IgG}$ antibodies to DENV with variable results. In general, performance of rapid devices improved 
between acute and convalescent sera and drawing of paired blood samples is suggested to improve accuracy of diagnosis (Nga et al., 2007). In this current study, 452 $(30.79 \%)$ cases were positive for Dengue or other forms of viral fever among the study population which reminds that Dengue is still a endemic disease of this part of the country. And out of 452 positive cases, Dengue NS1 Antigenemia was found in 32 out of 452 cases of clinically diagnosed Dengue infection which is positive in $7.08 \%$ of the these cases. This result is similar to the few studies conducted in South India (Nga et al., 2007). A total of 40/452 (8.85\%) cases were positive for NS1 alone or IgM alone or NS1 with IgM which were diagnosed as acute dengue infection. IgM detection between acute and convalescent sera, showed minimal improvement in sensitivity as compared to a marked improvement in specificity. Also, higher rates of positivity was reported in patients with Flavivirus infections, probably indicating cross reactivity with other viruses, especially Japanese encephalitis (JE) (Vaughn et al., 2000; Moorthy et al., 2009). When Compared with IgM, IgG shows poor sensitivity, but higher specificity. For both IgM and IgG detection, the likelihood ratios were not high enough to be recommended as a good stand alone diagnostic test. Testing with the device is relatively easy to perform, and interpretation of results, though interobserver Variation is rarely seen. In early infections and some secondary infection, detectable levels of $\operatorname{IgM}$ antibodies may be low. Some patients may not produce detectable levels of antibody within the first seven to ten day after infection. Where symptoms persist, patients should be retested 3-5 days after the first testing date. Serological cross - reactivity across the flavivirus group like Yellow fever virus, St. Louis encephalitis, West Nile virus, Japanese encephalitis are not uncommon. As with all diagnostic tests, all results must be correlated with other clinical findings. If the test result is negative and clinical symptoms persist, additional follow - up testing using other clinical methods is recommended. A negative result at any time does not exclude possibility of an early infection of dengue virus. Isolation of virus, antigen detection in fixed tissues, Indirect Immunofluorescence, RT-PCR and tests like hemagglutination inhibition (HAI) are more specific diagnosis method and it should be applied in order to confirm dengue virus infection as RCT is just a screening test. These are all the limitations of the current study.

In conclusion, the prevalence of Dengue fever in this study was found to be $8.85 \%$. Rapid Immuno chromatographic card tests are simple, feasible, cost-effective, less time consuming, with less labor involved, where sensitivity and specificity are good, but this card tests are only a screening test and so it cannot be used as a stand-alone test for diagnostic confirmation, but it could be supplemented with another diagnostic assays like ELISA for Dengue $\operatorname{IgM}$ and $\operatorname{IgM}$, Indirect Immuno fluorescence and Polymerase Chain Reaction for appropriate diagnosis of DENV infection. As Dengue has already emerged as a significant problem in our country, further population based studies are needed to evaluate the performance of the device in various hospital setups to know the epidemiology of Dengue infection in this part of the country.

\section{References}

Bharaj, P., Chahar, H.S., Pandey, A., Diddi, K., Dar, L., Guleria, R., et al. 2008. Concurrent infections by all four dengue virus serotypes during an outbreak of dengue in 2006 in Delhi, India. Virol. J., 5: 1 .

Blacksell, S.D., Newton, P.N., Bell, D., Kelley, J., Mammen, M.P. Jr, Vaughn, 
D.W., et al. 2006. The comparative accuracy of 8 commercial rapid immunochromatographic assays for the diagnosis of acute dengue virus infection. Clin. Infect. Dis., 42: 1127-34.

Cherian, T., Ponnuraj, E., Kuruvilla, T., Kirubakaran, C., John, T.J., Raghupathy, P. 1994. An epidemic of dengue haemorrhagic fever and dengue shock syndrome in and around Vellore. Indian J. Med. Res., 100: 51-6.

Gubler, D.J. 1998. Dengue and dengue hemorrhagic fever. Clin. Microbiol. Rev., 11: 480-96.

Gupta, E., Dar, L., Kapoor, G., Broor, S. 2006. The changing epidemiology of dengue in Delhi, India. Virol. J., 3: 92.

Guzman, M.G. 1996. Kourig Clinical \& Diagnostic Laboratory Immunology (1996) Vol. 3, No. 6, 621-627.

Monath, T.P. 1994. Dengue: The risk to developed and developing countries. Proc. Natl. Acad. Sci. USA, 91: 2395400.

Moorthy, M., S. Chandy, K. Selvaraj, A.M. Abraham. 2009. Evaluation of a Rapid Immuno-chromatographic device for rapid detection of $\mathrm{IgM}$ and $\mathrm{IgG}$ antibodies to Dengue viruses (DENV) in a tertiary care hospital in South India. Indian J. Med. Microbiol., 27(3): 254-6.

Myers, R.M., Carey, D.E., Rodriques, F.M. 1965. Experiences with the Isolation of Dengue Virus Types 1, 2 and 4 from Human Blood. Indian J. Med. Res., 53: 191-8.

Nga, T.T., Thai, K.T., Phuong, H.L., Giao, P.T., Hung le, Q., Binh, T.Q., et al.
2007. Evaluation of two rapid immunochromatographic assays for diagnosis of dengue among Vietnamese febrile patients. Clin. Vaccine Immunol., 14: 799-801.

Sathish, N., Manayani, D.J., Shankar, V., Abraham, M., Nithyanandam, G., Sridharan, G. 2002. Comparison of IgM capture ELISA with a commercial rapid immunochromatographic card test and IgM microwell ELISA for the detection of antibodies to dengue viruses. Indian J. Med. Res., 115: 31-6.

Singh, N.P., Jhamb, R., Agarwal, S.K., Gaiha, M., Dewan, R., Daga, M.K., et al. 2005. The 2003 outbreak of Dengue fever in Delhi, India. Southeast Asian J. Trop. Med. Public Health, 36: 1174-8.

Vaughn, D.W. et al., 2000. Dengue viremia titer, antibody response pattern, and virus serotype correlate with disease severity. J. Infect. Dis., 181: 2-9.

Vaughn, D.W., Nisalak, A., Kalayanarooj, S., Solomon, T., Dung, N.M., Cuzzubbo, A., Devine, P.L. 1998. Evaluation of a rapid immunochromatographic test for diagnosis of dengue virus infection. $J$. Clin. Microbiol., 36: 234-8.

Vijayakumar, T.S., Chandy, S., Sathish, N., Abraham, M., Abraham, P., Sridharan, G. 2005. Is dengue emerging as a major public health problem? Indian J. Med. Res., 121: 100-7.

Young, P.R., Hilditch, P.A., et al. 2008 J. Clin. Microbiol., Vol. 38, No.3, 10531057.

\section{How to cite this article:}

Gnana Prakash, K., R. Someshwaran and Anbu N. Aravazhi. 2016. Sero-Prevalence of Dengue Infection by Rapid Immunochromatographic Assay from Tertiary Care Medical College Hospital in Coimbatore, India. Int.J.Curr.Microbiol.App.Sci. 5(12): 106-111. doi: http://dx.doi.org/10.20546/ijcmas.2016.512.012 